\title{
Efecto del riego y la poda en el contenido de componentes menores y ácidos grasos del aceite de oliva virgen varietal Farga
}

\author{
Por J.I. Monteleone ${ }^{1,2, ~}{ }^{\otimes}$, C.A. Zuritz ${ }^{1,2}$, A.D. Gascón ${ }^{2}$ y J.A. Morábito ${ }^{2}$ \\ ${ }^{1}$ Consejo Nacional de Investigaciones Científicas y Técnicas (CONICET) \\ ${ }^{2}$ Facultad de Ciencias Agrarias. Universidad Nacional de Cuyo. Almirante Brown 500, \\ (5505) Chacras de Coria, Mendoza, Argentina \\ Correspondencia: jmonteleone@ conicet.gov.ar
}

\section{RESUMEN}

\begin{abstract}
Efecto del riego y la poda en el contenido de componentes menores y ácidos grasos del aceite de oliva virgen varietal Farga
\end{abstract}

Se analizó el efecto de distintas estrategias de riego y poda, sobre el contenido de componentes vinculados a la estabilidad oxidativa de los aceites de oliva vírgenes (polifenoles, clorofilas y carotenoides), variedad Farga. Se aplicaron cuatro tratamientos: T100\%SP (riego equivalente al 100\% de la demanda evapotranspiratoria (Etc) del cultivo durante todo el ciclo y sin poda invernal); T50\%SP (riego que equivalente al $50 \%$ de la Etc desde endurecimiento de carozo hasta cosecha y sin poda); T100\%CP (riego $100 \%$ y con poda); T50\%CP (riego deficitario y con poda). Se monitoreó el estado hídrico de las plantas a través del potencial hídrico del tallo a mediodía (PHT), el contenido de humedad del suelo y la radiación fotosintéticamente activa incidente en el interior de la copa de los olivos. El muestreo de aceitunas se extendió desde pre-envero hasta maduración completa. El aceite se extrajo en laboratorio por molienda de las aceitunas con molino de tornillo, termo-amasado, separación de fases sólida y líquida con una prensa manual y decantación para separar el aceite. Sobre el aceite extraído por métodos mecánicos se determinó: perfil de ácidos grasos, clorofilas y carotenoides (espectrofotometría a $670 \mathrm{~nm}$ y $472 \mathrm{~nm}$ ), polifenoles totales (absorbancia a $725 \mathrm{~nm}$ ) y ácidos grasos por cromatografía gaseosa capilar (CGC). Los datos de rendimiento graso (lipogénesis) expuestos muestran que no hay diferencias estadísticas entre un riego deficitario que cubre $50 \%$ de la Etc y el tratamiento control, tanto en plantas con poda o sin ella. El contenido de polifenoles, clorofilas y carotenoides en los aceites desciende a medida que avanza el estado de madurez de los frutos, independientemente del contenido inicial y el tratamiento. Los tratamientos con riego deficitario (T50\%) presentan contenidos más elevados de polifenoles (excepto para el muestreo de pre-envero), clorofilas y de carotenoides. No se observan diferencias estadísticamente significativas entre los tratamientos con y sin poda.

PALABRAS CLAVE: Aceite de oliva virgen - Olivos Poda - Polifenoles - Riego.

\section{SUMMARY}

Effect of irrigation and pruning on the amount of minor compounds and fatty acids in the virgin olive oils of the variety Farga

The effect of different irrigation and pruning strategies on the amount of substances related to the oxidative stability of virgin olive oils of the variety Farga (polyphenols, chlorophylls, carotenoids and fatty acids), was analyzed. Four treatments were used: T100\%SP (irrigation supplying 100\% of the plants' evaporative transpiration demand (Etc) during the entire cycle, without winter pruning); T50\%SP (irrigation supplying $50 \%$ of the plants' Etc from pit hardening to harvest, without winter pruning); T100\%CP (irrigation supplying $100 \%$, with pruning) and $\mathrm{T} 50 \% \mathrm{CP}$ (irrigation supplying $50 \%$, with pruning). The plants' hydric status was monitored through the stem hydric potential (SHP) at noon, and the soil moisture content and the photosynthetically active incident radiation within the tree branches were determined. The olive sampling was taken from pre-ripening to full maturity. The oil was extracted from the olives by grinding them in a laboratory screw type mill with thermo-kneading. The separation of solid and liquid phases was performed using a hand press and decanter to remove the oil. Chlorophylls and carotenoids (absorbance at $670 \mathrm{~nm}$ and $472 \mathrm{~nm}$ ) and total polyphenols (at $725 \mathrm{~nm}$ ), were determined by spectrophotometry on the mechanically extracted oil. Fatty acids were determined by capillary gas chromatography (CGC). Oil yield data show no statistical differences between deficit irrigation covering $50 \%$ of the ETc and the control treatment, both in plants with or without pruning. The contents of polyphenols, chlorophylls and carotenoids in the oils decrease with fruit ripening, regardless of the initial content and treatment. Deficit irrigation treatments (T 50\%) have higher contents of polyphenols (except for pre-envero sampling), chlorophylls and carotenoids. There are no statistically significant differences between treatments with and without pruning.

KEY-WORDS: Irrigation - Olive trees - Polyphenols Pruning - Virgin olive oil.

\section{INTRODUCCIÓN}

Las tendencias alimentarias internacionales están totalmente enfocadas a la generación de normativas que estandaricen y rijan las prácticas que aseguren la calidad e inocuidad de los alimentos que llegan al consumidor. En el inicio de la cadena agroalimentaria está la producción primaria agrícola que lentamente se va adaptando a las exigencias modernas de trazabilidad, con las denominadas Buenas Prácticas Agrícolas o BPA.

La estabilidad en los aceites de oliva vírgenes tiene una notable importancia desde el punto de vista comercial. Cuando se produce alteración, ésta se caracteriza por cambios físicos y químicos, 
descenso del valor nutricional y aparición de rancidez e incluso problemas de toxicidad. En el caso particular de los aceites de oliva mendocinos, se ha mencionado que sus bondades y buenos atributos de calidad se ven opacados por la variabilidad en su estabilidad oxidativa, que se refleja por la facilidad al deterioro oxidativo con formación de radicales superóxidos o peróxidos.

El aceite de oliva está formado principalmente por acilglicéridos; fracción que representa entre el $98,5 \%$ y el $99,5 \%$ del peso del aceite; con predominio de ácidos grasos mono y poliinsaturados que son los responsables de la inestabilidad oxidativa (Civantos L., 1999). El grupo de componentes minoritarios (hidrocarburos, esteroles, alcoholes triterpénicos, tocoferoles, fenoles, pigmentos, compuestos volátiles y aromáticos) tiene importancia desde el punto de vista del valor biológico del aceite, de sus características organolépticas y de su conservación. Polifenoles, tocoferoles y carotenoides presentes en el aceite de oliva son antioxidantes naturales que retrasan la oxidación.

La composición del aceite de oliva es la resultante de interacciones entre factores genéticos, ambientales y tecnológicos que marcan tanto la fase de desarrollo y de maduración del fruto como de su transformación (Montedoro y Servili, 1991). Por ello, resulta fundamental conocer la respuesta del olivo a diferentes estrategias de manejo, no sólo sobre la producción sino también sobre la calidad de productos manufacturados (Chova et al., 2000). Diversas experiencias muestran que los aceites de oliva obtenidos de olivares con riego deficitario controlado presentan mayor contenido de componentes antioxidantes, principalmente polifenoles (Servili et al., 2007). Se ha estudiado el efecto de la aplicación de diferentes niveles de riego a olivos sobre la actividad de la fenilalanina amonio liasa o PAL, enzima clave en el metabolismo fenólico, observándose una mayor actividad enzimática cuanto menor es el volumen de agua aplicada a los árboles (Patumi et al., 1998). En estudios similares, se observó que la aplicación de dosis crecientes de agua de riego a olivos jóvenes de la variedad Arbequina, tiene un claro efecto sobre el contenido de pigmentos fotosintéticos y de polifenoles del aceite. Es en los aceites correspondientes a los tratamientos con mayor aporte de agua, donde el contenido de polifenoles, pigmentos clorofílicos y carotenoides es notablemente inferior, lo que implica una disminución de la estabilidad de los aceites frente a la oxidación y un menor índice de amargor $\left(\mathrm{k}_{225}\right)$. En el extremo opuesto, los aceites de los tratamientos menos regados, presentan un elevado contenido de polifenoles y por lo tanto una mayor resistencia a la oxidación, pero con el inconveniente de resultar aceites amargos. Sobre la base de estos resultados y desde el punto de vista de la composición del aceite, los tratamientos más adecuados parecen encontrarse en las posiciones intermedias (Tovar De Dios, 2001). Varios trabajos sobre el efecto de estrategias de riego deficitario controlado (RDC), aplicadas duran- te el periodo estival, confirman una mayor estabilidad oxidativa en aceites provenientes de tratamientos de riego deficitarios (Faci et al., 2002; D’Andria et al., 2009).

Los datos disponibles sobre la relación entre la luz y los compuestos fenólicos, especialmente antocianos, flavonoides y derivados de ácidos hidroxicinámicos, indican que la luz es un factor determinante en su acumulación en especies vegetales. Esta relación se debe en parte a la regulación de la fotosíntesis, y de una manera más específica a las enzimas del metabolismo fenólico, especialmente la enzima PAL o fenilalaninaamonioliasa (Macheix et al., 1990). Por lo expuesto, la poda es una de las prácticas de cultivo que permitiría actuar sobre la iluminación y la aireación de la copa del olivo.

La composición en ácidos grasos del aceite y particularmente la relación entre los monoinsaturados y los poliinsaturados, influye en la estabilidad oxidativa del aceite y es un factor clave de la calidad nutricional del mismo (Uceda et al., 2005). Dicha composición acídica está influenciada por el cultivar y el medio agroecológico (Faci et al., 2002; Berenguer et al., 2006). En relación al cultivo, factores como la luz y la disponibilidad de agua, influyen en la biosíntesis de lípidos en los frutos (lipogénesis). Estudios realizados por Ortega (1962), Guerrero (1994). mostraron que el rendimiento en aceite de oliva, está relacionado con la posición de los frutos en la cubierta del árbol, observándose una mayor acumulación de aceite en aquellas olivas situadas en las zonas superiores de la copa del olivo, que recibían una mayor radiación solar, respecto a los situados en zonas internas del árbol y con menos luz. En relación con la disponibilidad de agua, cabe destacar que el carácter lineal del proceso de acumulación de aceite varía si los árboles se cultivan en condiciones limitantes y si se producen períodos de sequía durante el desarrollo del fruto, observándose un retraso en el proceso de acumulación de aceite (Lavee y Wodner, 1991; Pastor et al., 1996). Respecto a la composición acídica del aceite algunos autores no han detectado diferencias significativas en el contenido en ácidos grasos de los aceites en función del tratamiento de riego aplicado (Inglese et al., 1996; Patumi et al., 1999; Uceda, 2005), mientras que otros como Salas et al., (1997) y Faci et al., (2002) han observado un menor porcentaje de ácido palmitoleico y esteárico en aceites de secano. El riego, como práctica agrícola tiene una vinculación cuantitativa con la lipogénesis faltando evaluar si existe una interrelación cualitativa en forma dependiente.

El principal grupo de antioxidantes presente en la aceituna madura y en el aceite de oliva lo constituyen los compuestos fenólicos. Varios trabajos han relacionado el contenido en polifenoles con algunos parámetros utilizados para determinar la estabilidad de los aceites frente a la oxidación. Diferentes autores encontraron una correlación significativa entre los polifenoles totales y la estabilidad del aceite medida como índice de peróxidos (Montedoro et al., 1992; Beltrán et al., 2000). 
Los pigmentos presentes en el aceite de oliva se dividen en dos grupos: los pigmentos clorofílicos y los carotenoides. Una proporción de los pigmentos clorofílicos de la oliva se transforma en feofitinas. La feofitina «a» es el componente principal (44-58\% de los pigmentos totales), seguido por luteina (18-38\%) y $\beta$-caroteno (6-17\%) (Gandul-Rojas y Mínguez-Mosquera, 1996). La composición y el contenido total de pigmentos presentes de forma natural en el aceite de oliva, son importantes parámetros para la determinación de su calidad. Desde un punto de vista nutricional, la fracción carotenoide, principalmente el $\beta$-caroteno, constituye el factor pro-vitamina A.

En la actualidad se admite que la fotooxidación de los aceites vegetales, es inducida por las clorofilas y sus productos de degradación, las feofitinas. Pigmentos clorofílicos presentes en el aceite de oliva virgen son agentes fotosensibilizadores que se activan con la exposición a la luz, dando lugar a la formación de hidroperóxidos (Psomiadou y Tsimidou, 2002a). Pero en condiciones de oscuridad las clorofilas tienen una capacidad antioxidante que depende sensiblemente de su concentración en el aceite (Psomiadou y Tsimidou, 2002b).

El momento en que se recogen los frutos, y por tanto su índice de madurez, ejerce influencia en el contenido de pigmentos clorofílicos, carotenoides y tocoferoles de los aceites. El contenido de estos componentes desciende a medida que avanza el proceso de maduración (Motilva et al., 2008), al igual que el contenido de compuestos fenólicos totales que experimenta una marcada disminución desde envero hasta completa madurez de las aceitunas. (Cortesi y Rovellini, 2004).

Dada la importancia del contenido de polifenoles, clorofilas y carotenoides en la estabilidad de los aceites y debido a que en la actualidad no hay estudios sistemáticos para las condiciones agroecológicas del oeste argentino (clima árido con menos de $200 \mathrm{~mm}$ anuales de precipitación y cultivos de regadío), que vinculen la influencia de prácticas agrícolas con el contenido de dichas componentes en los frutos y su transferencia a los aceites, se plantea en el presente trabajo, el objetivo de establecer el efecto de las prácticas agrícolas de riego y poda en el contenido de componentes vinculadas a la estabilidad oxidativa (polifenoles, clorofilas, carotenoides, ácidos grasos) del aceite virgen de oliva obtenido de la variedad Farga.

\section{MATERIALES Y MÉTODOS}

\subsection{Material vegetal}

El estudio se realizó para las campañas 2009 y 2010 en un monte comercial de olivos adultos (Olea europaea L.) cultivar 'Farga'. Geográficamente ubicado en Maipú (3301'S, 684ㅇ' O, 764 m.s.n.m.) en la provincia de Mendoza, Argentina, cuyas condiciones agroclimáticas se caracterizan por ser zona semiárida: isoyetas menores de
$250 \mathrm{~mm}$ anuales. La distancia de plantación es de 6 metros entre hileras y entre plantas. El suelo es de origen aluvial, de textura franco a franco arenoso alcanzando un $50 \%$ de pedregosidad a partir de los $60 \mathrm{~cm}$ de profundidad. El sistema de riego es por goteo con cuatro laterales por hilera. Los emisores son goteros integrados autocompensados, con un caudal de $1,6 \mathrm{~L} \mathrm{~h}^{-1}$ y la distancia entre ellos es de $80 \mathrm{~cm}$.

Se aplicaron cuatro tratamientos, resultantes de la combinación de dos estrategias de riego y dos estrategias de poda. Las condiciones de riego fueron: $\mathrm{T} 100 \%$, un tratamiento control que se regó reponiendo el $100 \%$ de la demanda evapotranspiratoria del cultivo (Etc) durante todo el ciclo y $\mathrm{T} 50 \%$, un tratamiento de riego deficitario controlado donde se repuso el $50 \%$ de la Etc a partir de endurecimiento de carozo (principio de enero) y hasta cosecha (el resto del ciclo se regó cubriendo el $100 \%$ de la Etc). Ambos tratamientos de riego se aplicaron bajo dos condiciones de poda: plantas sin podar (SP) y plantas con una poda invernal de raleo (CP).

El diseño experimental fue de bloques al azar con cuatro repeticiones. Las parcelas experimentales están formadas por cuatro árboles continuos en la hilera (dos extremos como bordes y los dos centrales como unidades de medida).

\subsection{Programación del riego}

El riego se aplicó «a tiempo real» reponiendo los consumos diarios estimados (Etc). El cálculo de las necesidades de riego se realizó utilizando la metodología propuesta por Pizarro (1996). La evapotranspiración del cultivo de referencia (Eto) se obtuvo de la estación meteorológica oficial más cercana que utiliza para su estimación la ecuación de Penman-Monteith modificación FAO (Allen et al., 1998). Los valores de coeficiente de cultivo (Kc) se estimaron según la metodología que recomienda FAO para olivos con un $40-60 \%$ de cobertura de suelo por el dosel y con una altura máxima entre 3 y 5 metros. Los valores de Kc para las distintas etapas del cultivo fueron: $\mathrm{Kc}$ inicial $=0.65$; $\mathrm{Kc}$ medio $=0.70$ y Kc final $=0.70$. Estos valores se corrigieron según las condiciones agroclimáticas locales con la fórmulas referenciadas.

En las plantas con tratamiento de riego deficitario se obturó el $50 \%$ de los goteros que irrigaban cada planta, con cintas plásticas «tapagoteros» a fin de lograr el déficit deseado.

\subsection{Medida del potencial hídrico de la planta y humedad del suelo}

A fin de evaluar la respuesta de la planta a las dos estrategias de riego, se midió semanalmente el potencial hídrico xilemático al mediodía. Se utilizó una cámara de presión o cámara de Scholander modelo 4.0 Biocontrol.

Para monitorizar el estado hídrico del suelo se extrajeron muestras de suelo de cada tratamiento 
de riego a fin de determinar humedad gravimétrica. Las muestras se extrajeron entre los 30 y $50 \mathrm{~cm}$ de profundidad debido a que el elevado porcentaje de pedregosidad $(50 \%)$ impidió barrenar a mayor profundidad.

\subsection{Medida de la radiación fotosintéticamente activa (PAR)}

Se midió la radiación incidente en el interior de la copa del árbol con un ceptómetro con sensor tipo quantum Cavadevices, el cual integra el flujo recibido en un metro lineal $\left(\mu \mathrm{mol} \mathrm{m} \mathrm{m}^{-2} \mathrm{~s}^{-1}\right)$. Las medidas se realizaron en las caras norte y sur de la copa del árbol y a 1,7 $\mathrm{m}$ de altura.

\subsection{Muestreo de frutos}

La recolección de aceitunas se realizó desde pre-envero hasta completa maduración. Se extrajeron muestras de aceitunas en 4 oportunidades (22 marzo, 06 abril, 20 abril y 6 mayo) considerando un período de maduración de 50 días. Las muestras estuvieron constituidas por 3 kilogramos de aceitunas por cada tratamiento y repetición.

\subsection{Extracción de aceite}

Las aceitunas se sometieron a molienda con molino de tornillo de laboratorio. La pasta obtenida se sometió a termo-batido y la separación de fases sólida y líquida se realizó con una prensa manual. El mosto oleoso se decantó para separar el aceite y posteriormente se filtró. Los aceites se conservaron en frascos de vidrio color caramelo cerrados con barrido de nitrógeno, a una temperatura de $6{ }^{\circ} \mathrm{C}$ hasta su análisis.

En cada oportunidad de muestreo se determinó Índice de Madurez de los frutos según la metodología descrita por la Estación de Olivicultura y Elaiotecnia de Jaén (Hermoso et al., 1991) y rendimiento graso por soxhlet, método oficial del Código Alimentario Argentino (SAGPyA, 2009).

\subsection{Perfil de ácidos grasos}

El perfil de ácidos grasos se determinó por cromatografía gaseosa capilar (CGC) según el método propuesto por el Consejo Oleícola Internacional (2001), «Preparación de los ésteres metílicos de ácidos grasos del aceite de oliva y del aceite de orujo de oliva» y para la metilación el método que se describe en la Norma ISO 5509:2000(E) cláusula 5 (Método de trans-esterificación). La cuantificación de los ácidos grasos se realizó por el método de normalización interna. Se utilizó un cromatógrafo de gases marca Hewlett Packard 5890 Serie II plus, con detector FID. Las condiciones cromatográficas fueron: Columna HP-INNO WAX (30 m), Detector FID, Carrier $\mathrm{N}_{2}$, Vol. Inyectado 2,00 LI, Flujo: $1,10 \mathrm{~mL} \mathrm{~min}{ }^{-1}$, temperatura inyección: $200^{\circ} \mathrm{C}$, tem- peratura columna: Prog. $130^{\circ} \mathrm{C}$ a $220^{\circ} \mathrm{C}$ y temperatura detector: $260^{\circ} \mathrm{C}$ (FID).

\subsection{Clorofilas y carotenoides en aceite}

La valoración global del contenido en pigmentos clorofílicos y carotenoides se ha realizado siguiendo el método propuesto por Mínguez et al., (1991). Se basa en determinar la absorbancia de una disolución de aceite de oliva a la longitud de onda de máxima absorción del componente mayoritario, es decir, la feofitina a y la luteína respectivamente. La fracción carotenoide se cuantifica mediante la absorbancia a $470 \mathrm{~nm}$ y la fracción clorofílica a $670 \mathrm{~nm}$. La concentración de pigmentos clorofílicos y carotenoides se realiza aplicando la siguiente fórmula (Papaseit, 1986): $C=\left[\left(E^{*} V_{f}\right) /\left(E_{1 \%}{ }^{*} p\right)\right]^{\star} 10000$, donde: $\mathrm{C}=$ Concentración (mg de clorofilas o carotenos $/ \mathrm{kg}$ de aceite), $\mathrm{V}_{\mathrm{f}}=$ Volumen final del extracto de pigmentos $(\mathrm{ml}) \mathrm{p}=$ Peso de la muestra de aceite (g), $E_{1 \%}=$ Absorbancia específica de una disolución al $1 \%$ medida en una cubeta de $1 \mathrm{~cm}\left(\mathrm{E}_{1 \%}\right.$ luteína $a=2000$ y $E_{1 \%}$ feofitina $\left.=613\right)$. $E=$ Absorbancia $a$ la longitud de onda de medida.

\subsection{Polifenoles totales}

La determinación de los polifenoles totales se realizó por espectrofotometría UV-Visible de acuerdo con el método descrito por Vázquez et al. (1973). Se introduce como modificación la utilización del reactivo Folin-Ciocalteau (Beltrán et al., 1995).

\subsection{Análisis de datos}

El análisis se realizó con el software estadístico Infostat $₫$ versión 2008 . El análisis de la varianza se realizó con un nivel de significación del 95 \% para los parámetros analizados. Cuando las diferencias eran significativas, se ha aplicado un test de comparación de medias LSD.

\section{RESULTADOS Y DISCUSIÓN}

\subsection{Riego}

En la Tabla 1 se presenta la lámina total recibida (riego + precipitación efectiva) en ambos tratamientos de riego durante el ciclo del cultivo. La lámina de agua recibida por el tratamiento de riego control (T100\%) fue de $653 \mathrm{~mm}$ mientras que el tratamiento de riego deficitario (T50\%) recibió 492 $\mathrm{mm}$. Si se considera el total de agua recibida, el tratamiento con riego deficitario recibió $75 \%$ del agua del tratamiento control.

En la tabla 2 se observa que los valores promedio de la humedad gravimétrica del suelo y del potencial xilemático (PHT), durante todo el período de restricción presentaron diferencias significativas entre ambos tratamientos de riego. No se observó 
Tabla 1

Agua total (lámina de riego y precipitación efectiva) recibida en los tratamientos

de riego

\begin{tabular}{|c|c|c|c|c|c|c|}
\hline \multicolumn{7}{|c|}{ Cantidad de agua recibida (mm) } \\
\hline \multirow[t]{2}{*}{ Tratamiento } & \multicolumn{2}{|c|}{$\begin{array}{c}\text { Previo al riego } \\
\text { deficitario } \\
(09 / 2009-01 / 2010)\end{array}$} & \multicolumn{2}{|c|}{$\begin{array}{c}\text { Período de riego } \\
\text { deficitario } \\
(01 / 2010-05 / 2010)\end{array}$} & \multicolumn{2}{|c|}{ Total } \\
\hline & Riego & $P p e^{1}$ & Riego & Ppe & Riego + Ppe & $\%$ \\
\hline T100\% Etc & 301 & 0 & 323,0 & 28,8 & 652,8 & 100,0 \\
\hline T50\% Etc & 301 & 0 & 161,7 & 28,8 & 491,5 & 75,3 \\
\hline
\end{tabular}

${ }^{1} P$ pe: precipitación efectiva

efecto de la poda. Los valores de humedad gravimétrica corresponden a los primeros $40 \mathrm{~cm}$ del perfil del suelo debido a que la elevada pedregosidad no permitió la extracción a mayor profundidad, por lo que no se pudo determinar el contenido de humedad próximo a la profundidad efectiva de raíces $(80 \mathrm{~cm})$. La humedad en los tratamientos con riego deficitario (T50\%) osciló entre 10 y $13 \mathrm{~g} \% \mathrm{~g}$, mientras que para el riego control (T100\%) osciló entre 20 y $21 \mathrm{~g} \% \mathrm{~g}$. Los valores promedio del PHT oscilaron entre $-1,72$ y $-1,78 \mathrm{MPa}$ para el riego control y de -2,66 a -2,90 MPa para el riego deficitario.

En la Figura 1 puede observarse la evolución del potencial hídrico xilemático a mediodía (PHT) desde el inicio de la aplicación del tratamiento durante el ciclo 2009-2010. Desde el inicio de la restricción hídrica y durante casi todo este período se presentaron diferencias entre tratamientos de riego, tanto bajo condiciones de poda como en las plantas sin podar. Las diferencias entre tratamien-
Tabla 2

Humedad gravimétrica del suelo en el perfil a $40 \mathrm{~cm}$ de profundidad y potencial hídrico (PHT). Valores promedio del período de restricción

\begin{tabular}{ccc}
\hline Tratamiento & Humedad $(\mathbf{g} \% \mathbf{g})$ & $\begin{array}{c}\text { PHT } \\
\mathbf{( M P a})\end{array}$ \\
\hline T100\% SP & $20,04 \pm 2,36 \mathrm{a}^{1}$ & $-1,72 \pm 0,20 \mathrm{a}$ \\
T50\% SP & $10,16 \pm 2,16 \mathrm{~b}$ & $-2,90 \pm 0,64 \mathrm{~b}$ \\
T100\% CP & $21,27 \pm 1,99 \mathrm{a}$ & $-1,78 \pm 0,21 \mathrm{a}$ \\
T50\% CP & $13,24 \pm 3,43 \mathrm{~b}$ & $-2,66 \pm 0,65 \mathrm{~b}$ \\
\hline
\end{tabular}

${ }^{1}$ Distintas letras indican diferencias significativas $(p \leq 0,05)$.

tos disminuyeron en dos momentos que coinciden con las dos precipitaciones más importantes que se registraron (3 de febrero y 19 de marzo). Los valores de $\mathrm{PHT}$ llegaron a $-3,8 \mathrm{MPa}$ con el riego deficitario, mientras que en el tratamiento control

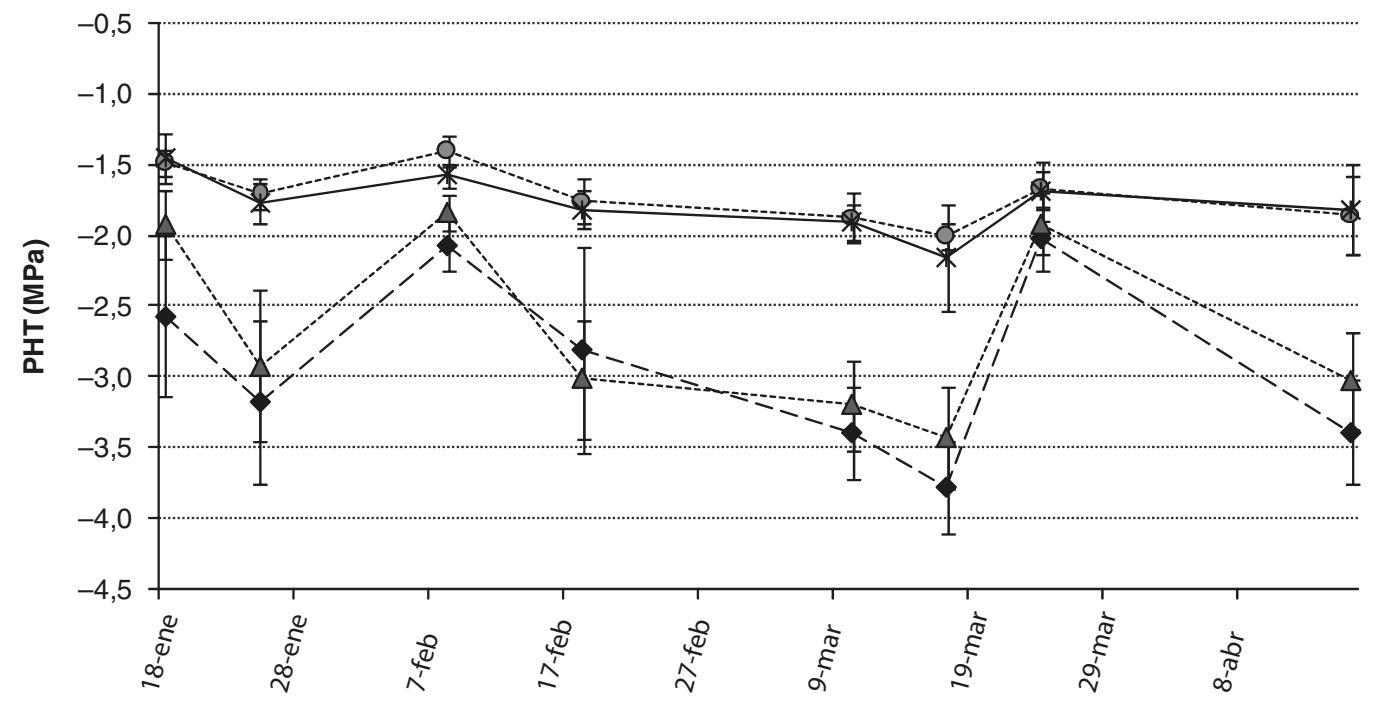

Fecha

\begin{tabular}{|c|c|c|c|}
\hline - o- T100\% SP & $-\mathrm{T} 50 \% \mathrm{SP}$ & * & $\cdots \triangle-\cdots$ T $50 \%$ CP \\
\hline
\end{tabular}

Figura 1

Variación del potencial hídrico de tallo $(\mathrm{PHT})$ al mediodía durante el período de riego deficitario. Cada valor de PHT representa la media de 8 mediciones. Barras verticales representan la desviación estándar. 
los valores de PHT se mantuvieron entre $-1,5$ y $-2 \mathrm{MPa}$. Los frutos en las plantas con riego deficitario mostraron deshidratación en reiterados momentos del período de restricción.

Los resultados confirman que el PHT mediodía es un buen indicador del estado hídrico en plantas de olivo. Tal aseveración coincide con trabajos de otros autores (Moriana et al., 2002). Este indicador evidenció el estrés de las plantas bajo tratamiento de riego deficitario controlado.

\subsection{Medida de radiación fotosintéticamente activa (PAR)}

La tabla 3 muestra el efecto de la poda invernal en el porcentaje de radiación incidente en el interior de la copa de los árboles. Las mediciones se realizaron desde el inicio de la fructificación y hasta cosecha. La poda aumentó el porcentaje de radiación incidente (PAR) con diferencias estadísticamente significativas entre tratamientos con y sin poda, no se observó efecto de los tratamientos de riego.

\subsection{Humedad y rendimiento graso}

En la tabla 4 se observan los valores de humedad y grasa total de las aceitunas durante el proceso de maduración. El contenido de humedad de los frutos es menor en los tratamientos con riego defi- citario en la última etapa de madurez. Los datos de rendimiento graso muestran que no hay diferencias estadísticas entre tratamientos, lo cual permite sugerir el ahorro de agua de riego sin perjudicar los rendimientos industriales en la obtención de aceites vírgenes de oliva.

\subsection{Contenido de polifenoles, clorofilas y carotenoides}

En las figuras 2, 3 y 4 se observa la evolución del contenido de sustancias antioxidantes de los aceites a lo largo del proceso de maduración. El contenido de polifenoles, clorofilas y carotenoides desciende a medida que avanza el estado de madurez de los frutos, independientemente del contenido inicial y el tratamiento aplicado. Los tratamientos con riego deficitario presentan contenidos más elevados de polifenoles (excepto para el muestreo de pre-envero), clorofilas y de carotenoides en los aceites (con diferencias significativas). No se observan diferencias estadísticamente significativas entre los tratamientos con y sin poda.

Las concentraciones de clorofilas y carotenoides en los aceites y su evolución a lo largo del proceso de maduración coinciden con lo observado por Criado-García et al. (2007) para la cultivar «Farga». La disminución del contenido de clorofilas es mayor (entre el $95 \%$ y el $97 \%$ ) que en el caso de los carotenoides (entre $81 \%$ y el $88 \%$ ).

Tabla 3

Porcentaje de radiación fotosintéticamente activa incidente en la copa del olivo

\begin{tabular}{ccccc}
\hline Ciclo 2009-2010 & \multicolumn{4}{c}{ Porcentaje de radiación PAR incidente (\%) } \\
\hline Fecha & T100\% SP & T50\% SP & T100\% CP & T50\% CP \\
\hline 13/01/2010 & $11,05 \mathrm{a}$ & $10,23 \mathrm{a}$ & $14,28 \mathrm{a}$ & $21,78 \mathrm{a}$ \\
30/01/2010 & $11,47 \mathrm{a}^{1}$ & $13,01 \mathrm{a}$ & $15,51 \mathrm{ab}$ & $21,62 \mathrm{~b}$ \\
$13 / 02 / 2010$ & $08,62 \mathrm{a}$ & $13,82 \mathrm{ab}$ & $16,64 \mathrm{ab}$ & $19,31 \mathrm{~b}$ \\
28/02/2010 & $13,19 \mathrm{a}$ & $13,38 \mathrm{a}$ & $21,18 \mathrm{~b}$ & $22,79 \mathrm{~b}$ \\
$11 / 04 / 2010$ & $11,15 \mathrm{a}$ & $09,57 \mathrm{a}$ & $18,48 \mathrm{~b}$ & $21,02 \mathrm{~b}$ \\
\hline
\end{tabular}

${ }^{1}$ Distintas letras indican diferencias significativas $(p \leq 0,05)$.

Tabla 4

Efecto del riego y la poda sobre el porcentaje de humedad y contenido graso de las aceitunas

\begin{tabular}{|c|c|c|c|c|c|c|c|c|}
\hline \multirow{2}{*}{$\begin{array}{c}\text { Índice } \\
\text { madurez }\end{array}$} & \multicolumn{2}{|c|}{ T100\% CP } & \multicolumn{2}{|c|}{ T50\% CP } & \multicolumn{2}{|c|}{ T100\% SP } & \multicolumn{2}{|c|}{ T50\% SP } \\
\hline & Humedad & $\begin{array}{l}\text { Grasa } \\
\text { total }^{1}\end{array}$ & Humedad & $\begin{array}{l}\text { Grasa } \\
\text { total }^{1}\end{array}$ & Humedad & $\begin{array}{l}\text { Grasa } \\
\text { total }^{1}\end{array}$ & Humedad & $\begin{array}{l}\text { Grasa } \\
\text { total }^{1}\end{array}$ \\
\hline 1,50 & 61,94 & 13,78 & 62,64 & 12,61 & 62,35 & 13,22 & 63,38 & 12,60 \\
\hline 2,12 & 59,74 & 15,19 & 59,51 & 14,27 & 60,67 & 14,13 & 57,48 & 14,00 \\
\hline 3,84 & $59,43 a^{2}$ & 17,17 & $58,40 a b$ & 16,48 & $60,90 \mathrm{a}$ & 16,22 & $56,34 \mathrm{~b}$ & 16,09 \\
\hline
\end{tabular}

Valores expresados en \%

${ }^{1}$ Sobre peso húmedo.

${ }^{2}$ Distintas letras indican diferencias significativas $(p \leq 0,05)$. 




Efecto de los tratamientos de riego y poda en la evolución de los polifenoles totales (ppm ácido cafeico), en los aceites de oliva. Barras verticales representan la desviación estándar.

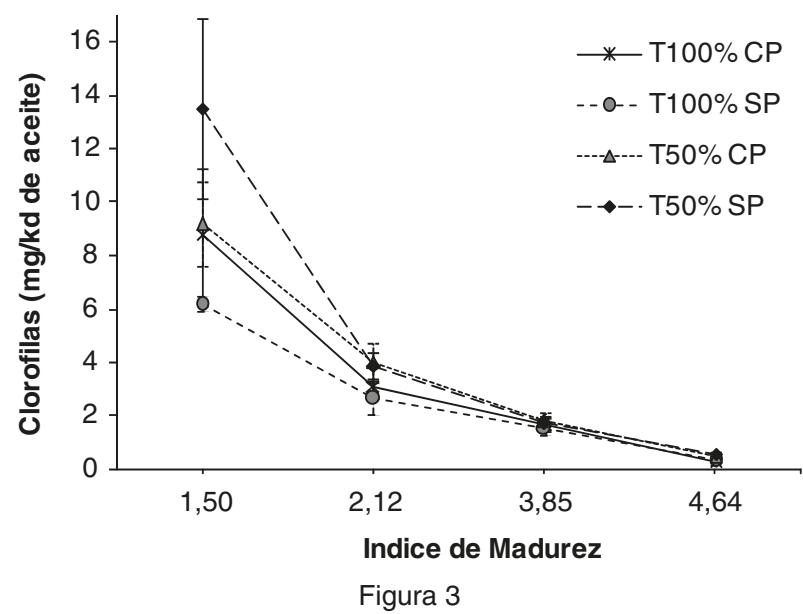

Efecto de los tratamientos de riego y poda en la evolución de las clorofilas ( $\mathrm{mg} / \mathrm{kg}$ de aceite) en los aceites de oliva vírgenes. Barras verticales representan la desviación estándar.

\subsection{Perfil de ácidos grasos}

En la composición acídica de los de los aceites se observan diferencias estadísticas significativas en el contenido de los ácidos palmitoleico, hepta-

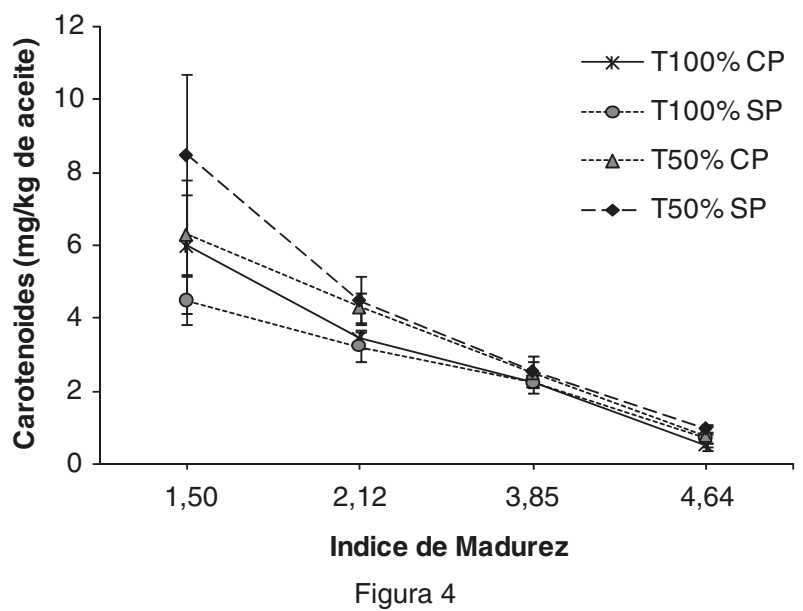

Efecto de los tratamientos de riego y poda en la evolución de los carotenoides ( $\mathrm{mg} / \mathrm{kg}$ de aceite) en los aceites de oliva vírgenes. Barras verticales representan la desviación estándar.

decanoico y esteárico, entre los tratamientos con distinta condición de riego. Los tratamientos con riego deficitario presentan menores contenidos de estos ácidos. Estos resultados coinciden con trabajos de otros autores como Salas et al., (1997) y Faci et al., (2002), que han observado un menor porcentaje de ácido palmitoleico y esteárico en aceites de olivos en secano. Servili et al. (2007) encontró un menor contenido de ácido palmitoleico en aceites provenientes de olivos con riego deficitario y un mayor contenido de ácido linoleico en estos aceites.

\section{CONCLUSIONES}

Los datos de rendimiento graso muestran que no hay diferencias estadísticas entre un riego deficitario que cubre $50 \%$ de la Etc y un tratamiento que repone el $100 \%$ de la Etc, tanto en plantas con poda o sin ella. Estos resultados nos sugieren que se pueden aplicar estrategias de riego deficitario controlado al cultivo, permitiendo un ahorro de agua de riego sin perjudicar los rendimientos industriales en la obtención de aceites vírgenes de oliva.

Tabla 5

Perfil de ácidos grasos (\%) del aceite varietal Farga, en relación a los tratamientos de riego y poda

\begin{tabular}{|c|c|c|c|c|c|c|c|c|}
\hline Tratamiento & $16: 0$ & $16: 1$ & $17: 0$ & $18: 0$ & $18: 1$ & $18: 2$ & $18: 3$ & 20:0 \\
\hline T100\% CP & $\begin{array}{c}14,75 \\
\pm 0,17 a\end{array}$ & $\begin{array}{l}1,43 \\
\pm 0,07 a^{1}\end{array}$ & $\begin{array}{c}0,10 \\
\pm 0,00 \mathrm{a}\end{array}$ & $\begin{array}{c}1,54 \\
\pm 0,03 a\end{array}$ & $\begin{array}{c}70,28 \\
\pm 0,49 a\end{array}$ & $\begin{array}{c}9,63 \\
\pm 0,42 \mathrm{a}\end{array}$ & $\begin{array}{c}0,81 \\
\pm 0,06 \mathrm{a}\end{array}$ & $\begin{array}{c}0,34 \\
\pm 0,01 \mathrm{a}\end{array}$ \\
\hline $\mathrm{T} 50 \% \mathrm{CP}$ & $\begin{array}{c}14,70 \\
\pm 0,35 \mathrm{a}\end{array}$ & $\begin{array}{c}1,29 \\
\pm 0,15 b\end{array}$ & $\begin{array}{c}0,12 \\
\pm 0,01 \mathrm{~b}\end{array}$ & $\begin{array}{c}1,62 \\
\pm 0,04 a b\end{array}$ & $\begin{array}{c}69,42 \\
\pm 0,66 \mathrm{a}\end{array}$ & $\begin{array}{c}10,25 \\
\pm 0,91 \mathrm{a}\end{array}$ & $\begin{array}{c}0,85 \\
\pm 0,04 \mathrm{a}\end{array}$ & $\begin{array}{c}0,34 \\
\pm 0,01 \mathrm{a}\end{array}$ \\
\hline T100\% SP & $\begin{array}{c}14,92 \\
\pm 0,43 \mathrm{a}\end{array}$ & $\begin{array}{c}1,52 \\
\pm 0,12 \mathrm{a}\end{array}$ & $\begin{array}{c}0,10 \\
\pm 0,00 \mathrm{a}\end{array}$ & $\begin{array}{c}1,47 \\
\pm 0,12 \mathrm{a}\end{array}$ & $\begin{array}{c}69,78 \\
\pm 0,83 \mathrm{a}\end{array}$ & $\begin{array}{c}9,78 \\
\pm 0,42 \mathrm{a}\end{array}$ & $\begin{array}{c}0,82 \\
\pm 0,03 \mathrm{a}\end{array}$ & $\begin{array}{r}0,33 \\
\pm 0,01 \mathrm{a}\end{array}$ \\
\hline T50\% SP & $\begin{array}{c}14,24 \\
\pm 0,29 a\end{array}$ & $\begin{array}{c}1,14 \\
\pm 0,07 \mathrm{~b}\end{array}$ & $\begin{array}{c}0,13 \\
\pm 0,01 \mathrm{~b}\end{array}$ & $\begin{array}{c}1,73 \\
\pm 0,15 \mathrm{~b}\end{array}$ & $\begin{array}{c}70,07 \\
\pm 0,76 \mathrm{a}\end{array}$ & $\begin{array}{c}10,45 \\
\pm 0,58 \mathrm{a}\end{array}$ & $\begin{array}{c}0,83 \\
\pm 0,03 \mathrm{a}\end{array}$ & $\begin{array}{c}0,34 \\
\pm 0,02 \mathrm{a}\end{array}$ \\
\hline
\end{tabular}

16:0, ácido palmítico; 16:1, ácido palmitoleico; 17:0, ácido heptadecanoico; 18:0, ácido esteárico; 18:1, ácido oleico; 18:2, ácido linoleico; 18:3, ácido linolénico; 20:0, ácido araquídico.

${ }^{1}$ Valores seguidos de distinta letra representan diferencias significativas $(p \leq 0,05)$ 
En relación al contenido de sustancias antioxidantes presentes en los aceites de oliva que dan estabilidad a la oxidación, se observa que los riegos deficitarios incrementan, con diferencias estadísticas, el contenido de polifenoles, clorofilas y carotenoides.

\section{AGRADECIMIENTOS}

CONICET, Argentina; Secretaría de Ciencia Técnica y Posgrado, Universidad Nacional de Cuyo, Mendoza, Argentina; Establecimiento Olivícola Palazzini, Maipú, Mendoza, Argentina.

\section{REFERENCIAS}

Allen R, Pereira L, Raes D, Smith M. 1998. Crop evapotranspiration guidelines for computing crop water requirements. FAO, Irrigation and Drainage 56. Rome, Italy.

Beltrán G, Jiménez A, Uceda M. 1995. Efecto del régimen hídrico del cultivo sobre la fracción fenólica del aceite de oliva de la variedad Arbequina. I Simposio del olivo

Arbequino en Cataluña, Borges Blanques, págs. 153155.

Beltrán G, Jiménez A, Aguilera M, Uceda M. 2000. Análisis mediante HPLC de la fracción fenólica del aceite de oliva virgen de la variedad Arbequina. Relación con la medida del amargor K225 y la estabilidad. Grasas Aceites 51, 320-324

Berenguer M, Vossen P, Grattan S, Connell J, Polito V. 2006. Tree irrigation level for optimum chemical and sensory properties of olive oil. Hortscience 41, 427432.

Civantos López-Villalta L. 1999. Obtención del Aceite de Oliva Virgen. Ed. Agrícola Española S.A., España.

Chova M M, Peña F, Del Campillo MC, Delgado A, Díaz M. 2000. Efecto de la corrección de la clorosis férrica en olivar con fosfato de hierro en los parámetros de calidad del aceite de oliva virgen. Edafología 7, 9-15.

Consejo Oleícola Internacional. 2001. Preparación de los ésteres metílicos de los ácidos grasos del aceite de oliva y el aceite de orujo de oliva. COI/T20/Documento № 24

Cortesi N, Rovellini P. 2004. Estado oxidativo del aceite de oliva virgen: efectos de los antioxidantes naturales. Olivae 101, 28-33.

Criado MN, Motilva M, Goñi M, Romero MP. 2007. Comparative study of the maturation process of the olive frui ton the chlorophyll and carotenoid fractions of drupes and virgin oils Arbequina and Farga cultivars. Food Chem. 100, 748-755.

D'Andria R, Lavini A, Morelli G, Sebastiani L, Tognetti R. 2009. Physiological and productive responses of Olea europea L. cultivars Frantoio and Leccino to a regulated irrigation regime. Plant Biosyst. 143, 222 -231

Faci J, Berenguer M, Espada J, Gracia S. 2002. Effect of variable water irrigation supply in olive (olea europaea I.) cv. arbequina in Aragon (Spain). II. Extra virgin oil quality parameters. Acta Hortic. (ISHS) 586, 649-652.

Gandul-Rojas B, Mínguez-Mosquera M.I. 1996 Chlorophyll and carotenoid composition in virgin olive oils from various Spanish olive varieties. J. Sci. Food Agric. 72, 31-39.
Guerrero, J. 1994. Nueva Olivicultura. Editorial MundiPrensa, Madrid.

Hermoso M, Uceda M, García A, Morales B, Frías M.L.; Fernández, A. 1991. Elaboración de aceite de oliva de calidad. Consejería de Agricultura y Pesca (Serie apuntes 5/92). Sevilla, España.

Inglese P, Barone E, Gullo G. 1996. The effect of complementary irrigation on fruit growth, ripening pattern and oil characteristics of olive (Olea europaea L.) cv. Carolea. J. Hort. Sci. 71, 257-263.

Lavee S, Wodner M. 1991. Factors affecting the nature of oil accumulation in fruit of olive (Olea europaea L.) cultivars. J. Hortic. Sci. 66, 583-591.

Macheix JJ, Fleuriet A, Billot J. 1990. Fruit Phenolics. CRC Press, Boca Raton, Florida.

Mínguez-Mosquera MI, Rejano-Navarro L, Gandul-Rojas B, Sanchez-Gomez AH, Garrido-Fernandez J. 1991. Color- Pigment Correlation in Virgen Olive Oil. J. Am. Oil Chem. Soc. 68, 332-336.

Montedoro G, Servili M. 1991. Chimica e qualità dell' olio di oliva: i fattori che la condizionano. Atti Convegno L' olio di oliva ed il suo futuro, Spoleto, págs. 33-55.

Montedoro G, Servili M, Baldiolo M, Miniati E. 1992. Simple and hydrolizable phenolics compounds in virgin olive oil. Their extraction, separation and quantitave and semiquantitave evaluation by HPLC. J. Agric. Food Chem. 40, 1571-1576.

Moriana A, Fereres E. 2002. Plant indicators for scheduling irrigation of young olive tree. Irrigation Science 21, 83-90.

Motilva MJ, Criado MN, Romero MP, Casanovas M. 2008. Pigment profile and colour of monovarietal virgin olive oils from Arbequina cultivar obtained during two consecutivw crop seasons. Food Chem. 110, 873-880.

Ortega Nieto JM. 1962. La poda del olivo. Ministerio de Agricultura, Madrid.

Papaseit J. 1986. El color del aceite de oliva extra virgen, característica de calidad. Grasas Aceites 37, 204206.

Pastor M, Castro J, Manrique T, Gómez L. 1996. Crecimiento del fruto, formación de aceite y producción del olivar regado con diferentes programas de riego por goteo en Andalucía. Fruticultura Profesional 76, 6-23.

Patumi M, d'Andria R, Fontanazza G, Morelli G, Vergari G. 1998. Effetto dell' irrigazione sulla produzione e sullo sviluppo vegetativo di un giovane oliveto. Olivo\&Olio 1, 36-47.

Patumi M, d'Andria R, Fontanazza G, Morelli G, Giorio P, Sorrentino G. 1999. Yield and oil quality of intensively trained trees of three cultivars of olive (Olea europaea L.) under different irrigation regimes. J. Hortic. Sci. Biotech. 74, 729-737.

Pizarro, F. 1996. Riegos localizados de alta frecuencia. Goteo, microaspersión y exudación. Ediciones Mundi-Prensa, Madrid.

Psomiadou E, Tsimidou M. 2002 (a). Stability of Virgin Olive Oil. 1. Autoxidation Studies. J. Agric. Food Chem. 50, 716-721.

Psomiadou E, Tsimidou M. 2002 (b). Stability of Virgin Olive Oil. 2. Photo-oxidation Studies. J. Agric. Food Chem. 50, 722-727.

Salas J, Pastor M, Castro J, Vega V. 1997. Influencia del riego sobre la composición y características organolépticas del aceite de oliva. Grasas Aceites 48, 7482.

Secretaría de Agricultura, Ganadería y Pesca. 2009. Marco regulatorio. Código Alimentario Argentino, capítulo VII, artículo 520 a 552 tris. 
Servili M, Esposto S, Lodolini E, Selvaggini R, Taticchi A, Urbani S, Montedoro G, Serravalle M, Gucci R. 2007. Irrigation effects on quality, phenolic composition and selected volátiles of virgin olive oils cv. 'Leccino'. J. Agric. Food Chem. 55, 6609-6618

Tovar De Dios MJ, Motilva MJ, Romero MP 2001. Changes in the phenolic composition of virgin olive oil from Young tres (Olea europaea L. 'Arbequina') Grown under linear irrigation strategies. J. Agric. Food Chem. 49, 5502-5508.
Uceda M, Beltrán G, Jimenez A. 2005. Composición del aceite. En: Variedades de olivo en España (Libro II: Variabilidad y selección). Rallo, L. et al. (eds.). Junta de Andalucía, MAPA y Mundi-Prensa, Madrid, págs. 357-372.

Vázquez A, Janer C, Janer ML. 1973. Determinación de los polifenoles totales del aceite de oliva. Grasas Aceites 24, 350-355.

Recibido: 22/11/12 Aceptado: $18 / 2 / 13$ 